\title{
PASOS HACIA UNA NATURALIZACIÓN COGNITIVA EN LA FILOSOFÍA DEL DERECHO (INDEXICALIDAD CONSCIENTE E INCONSCIENTE: UNA REVISIÓN A LA EFICACIA NORMATIVA)*
}

\author{
STEPS TOWARDS A COGNITIVE NATURALIZATION IN LEGAL PHILO- \\ SOPHY (CONSCIOUS AND UNCONSCIOUS INDEXICALITY: A REVIEW \\ OF NORMATIVE EFFICACY)
}

\section{Enrique CÁCERES**}

Carlos Montemayor ${ }^{* * *}$

\section{Resumen:}

En la ciencia como en la filosofía es frecuente que, durante largos periodos, la actividad de sus respectivas comunidades centren su atención en un determinado problema o clase de problemas abordado(s) desde un cierto enfoque metodológico.

Las discusiones en el paradigma dominante en la filosofía del derecho no son una excepción y es así que desde hace ya mucho tiempo sus discusiones giran en torno a un núcleo de problemas abordados desde la metodología del análisis conceptual especulativo. El ejemplo paradigmático está representado por el problema de la relación entre derecho y moral.

Nuestra preocupación cambia el foco de atención y gira en torno a la idea de que una adecuada comprensión del concepto de eficacia jurídica (EJ) es una piedra de toque para comprender mejor el concepto del derecho. Muchas preguntas importantes pueden ser esclarecidas desde una respuesta adecuada a este concepto, entre ellas, la relativa a la relación entre derecho y moral. De manera específica nos interesa analizar preguntas del tipo ¿en qué sentido el derecho regula la vida social si la inmensa mayoría de sus des-

* Artículo recibido el 1o. de diciembre de 2014 y aceptado para su publicación el 22 de febrero de 2015.

** Instituto de Investigaciones Jurídicas de la UNAM, encacer@hotmail.com.

*** San Francisco State University, cmontema@sfsu.edu. 


\section{ENRIQUE CÁCERES / CARLOS MONTEMAYOR}

tinatarios desconocen las reglas del sistema?, ¿de qué manera determinan las decisiones de los operadores jurídicos si la determinación normativa es siempre una cuestión abierta y debatible?

Sin embargo, no únicamente cambiamos el foco de atención a un problema que ha sido poco atendido desde la filosofía del análisis conceptual especulativo. También proponemos un cambio metodológico, pues lo abordamos desde una particular concepción de la naturalización de la filosofía del derecho consistente en una continuación de los resultados arrojados por lo que ha dado en llamarse la nueva ciencia cognitiva. Ello implica que elevamos el estándar de justificación epistémica de la teoría jurídica más allá del análisis conceptual especulativo clásico.

Con base en ello, sometemos a revisión lo que damos en llamar las "idealizaciones cognitivas" que imperan acerca del derecho tanto en las intuiciones del hombre ordinario como en los teóricos de derecho provenientes de diversas tradiciones. El común denominador consiste en una sobrevaloración del papel del consciente y del control de la voluntad de los agentes en las conductas esperadas con base en el derecho. El hecho de que precisamente ese supuesto sea el centro de ataques de la nueva ciencia cognitiva y que no soporta el análisis de la investigación empírica nos lleva a tener que buscar otro punto de partida. Este lo encontramos en el situacionismo cognitivo, en la indexicalidad autoreferencial, así como en los sistemas 1 y 2 propuestos por de Kanheman.

El desplazamiento de nuestra preocupación a los agentes, más allá de las simples abstracciones conceptuales, al menos eso creemos, abre la puerta a nuevas investigaciones entre las cuales se encuentra la revisión de la tesis según la cual la eficacia del derecho presupone las operaciones no controladas conscientemente reveladas por la neuroética y la influencia que en ellas pueda tener la función constitutiva del leguaje normativo del derecho. En otras palabras, que existe una relación indisoluble entre derecho y moral a nivel cognitivo que constituye una condición de la existencia de los ordenamientos jurídicos.

\section{Palabras clave:}

Metodología jurídica, giro cognitivo, indexicalidad normativa, eficacia del derecho, naturalización de la filosofía del derecho. 


\begin{abstract}
:
\end{abstract}
In science and philosophy it is common that their communities concentrate, for long periods of time exclusively to a certain problem or set of problems guided by a particular methodological approach.

Same situation is present in legal philosophy. A dominant paradigm in legal methodology had led discussions to speculative conceptual analysis. $A$ very illustrative example of this is the debate regarding the relations between law and morality.

In our paper we take a different starting point and claim that an adequate understanding of legal efficacy is fundamental to address any issue regarding the concept of law. Crucial questions, including the relation between law and morality, become clearer when the efficacy of a legal system is explained. Specifically, we are interested in analyzing the following questions: In what sense is the law responsible for regulating society if the vast majority of its subjects do not know the rules of the system? And in what ways does the law determine the decisions of legal institutions if the content of law is always subject to an open debate?

We are not only shifting the focus of analysis to a set of problems that cannot be adequately understood by speculative conceptual analysis alone. We also argue for a change in methodology by taking a naturalistic approach to legal philosophy that engages with the most important findings in cognitive science in a meaningful way. In doing so, we examine what we call the "cognitive idealizations" that shape the intuitions of ordinary people and, to a large extent, of legal philosophers. The common denominator of these intuitions is a systematic overestimation of the role of conscious voluntary control in guiding the agents that are governed by the law. The fact that such conscious effortful control is one of the most salient targets of experimental criticism in recent cognitive science makes us consider alternative routes to approach the problem of legal efficacy, including work on situationism, indexicality, and Kahneman's distinction between systems 1 and 2. We believe that this theoretical reorientation towards agents and their cognitive capacities will transcend simple conceptual abstractions and open the path to new insights in legal philosophy, particularly with respect to the widely assumed thesis that the efficacy of legal norms requires explicit conscious reflection about norms and their consequences. An important conclusion of our arguments is that there must be a necessary cognitive relation between moral and legal reasoning.

\title{
Keywords:
}

Jurisprudential Methodology, Cognitive Turn, Normative Indexicality, Legal Efficacy, Naturalizing Jurisprudence. 
Sumario: I. Introducción. II. Pluralismo metódico, tradiciones en filosofía del derecho y situacionismo. III. Idealización cognitiva (el enfoque prescriptivo) vs. naturalización en la filosofía del derecho (el enfoque descriptivo como condición del prescriptivo). IV. La idealización cognitiva y el presupuesto de la eficacia jurídica en las distintas tradiciones filosóficojurídicas. V. Conclusión. VI. Bibliografía.

\section{INTRODUCCIÓN}

Los sujetos del derecho cumplen con normas muy diversas y complejas: leyes tributarias, penales, civiles y administrativas, entre muchas otras.

Los abogados, por su parte, deben dedicar varios años de su vida para comprender y conocer a fondo sólo un subconjunto de un sistema normativo.

Tomando en consideración esta importante diferencia entre juristas y no juristas no es una cuestión trivial saber cómo es posible que los sujetos de las normas logren obedecerlas sin tomar varios cursos de derecho y sin pensar constantemente en las implicaciones jurídicas de sus actos.

En este trabajo partimos del supuesto de que responder a esta pregunta requiere respuestas que van más allá de las hasta la fecha ofrecidas por la teoría del derecho y que para ello se requiere saber cuáles son los procesos cognitivos que hacen posible la eficacia normativa.

En la actualidad y dados los importantes avances en las ciencias cognitivas la teoría del derecho no puede ignorar esta pregunta cuya respuesta debe ser por lo menos compatible con los resultados de dichas ciencias.

En otras palabras, dado el nivel de avance de las últimas investigaciones cognitivas sobre la conducta humana resulta injustificado no tomar en serio en qué consiste la eficacia de los sistemas jurídicos $\mathrm{y}$, como ha sido hasta la fecha, conformarse con referir el concepto de pasada mediante elucubraciones especulativas. Muchas de las preguntas fundamentales planteadas por los teóricos del derecho dependen de la respuesta al problema de la eficacia normativa y son 
lo suficientemente relevantes como para no aprovechar las herramientas teóricas y conceptuales proporcionadas por la ciencia para intentar contestarla. Sólo por citar algunas consecuencias de esa respuesta vale señalar que de una adecuada explicación de la eficacia del derecho depende la cabal comprensión de la autoridad del derecho, de su legitimidad, de su incidencia o no en la configuración de prácticas sociales, de la justificación de la atribución de responsabilidad jurídica y la aplicación de sanciones o, incluso, siguiendo a Kelsen, la existencia misma de los sistemas jurídicos.

En este trabajo consideramos que para responder a esta pregunta central es necesario conocer qué procesos cognitivos son necesarios o suficientes para que la eficacia jurídica opere, respuesta que constriñe a las actuales teorías del derecho de modo tal que aquellas que no sean compatibles con los resultados de las ciencias resultarían inválidas o al menos relegadas al terreno de las explicaciones "metafísicas" en el sentido peyorativo otorgado por Carnap a la expresión. Asumimos, por lo tanto, que esta pregunta requiere considerar las respuestas que la ciencia cognitiva proporciona a la conducta humana, lo que ofrece una ruta hacia la naturalización del derecho: precisamente la que intentaremos seguir.

Por supuesto, apelar a la naturalización en la teoría del derecho no es del todo nuevo. De hecho, hay varias propuestas al respecto, algunas apelando al realismo de prácticas judiciales y los procesos epistémicos que éstas asumen, mientras que otras, en una zona de intersección con la neuroética apelan directamente a cuestiones neuronales o neurocientíficas.

Lo que es innovador en nuestro artículo son las siguientes tesis.

Proponemos que la indexicalidad cognitiva situada (relacionada con el problema del marco) puede ser consciente o inconsciente.

La evidencia relevante es la investigación de heurísticas para la decisión. La tesis fundamental que defendemos es que aun cuando la cognición consciente que permite la aplicación de las normas es, obviamente, suficiente para el seguimiento del orden normativo por parte de los operadores jurídicos, dicho tipo de cognición no es necesaria para la eficacia general del sistema. Es sólo necesaria para jueces, abogados y aquellos que deben seguir explícitamente la relación entre preceptos legales y casos concretos. Aunque la cognición cons- 
ciente es suficiente para el seguimiento de normas, ésta no ofrece un modelo realista de la eficacia normativa: uno jamás piensa en las regulaciones sobre compra-venta cuando paga un boleto del metro.

La indexicalidad situada inconsciente y no basada en razonamiento inferencial explícito es necesaria para navegar exitosamente contextos epistémicos y morales. Estos son utilizados por la mayoría de los sujetos normativos, sin necesidad de formular explícitamente aplicaciones de preceptos a su situación concreta. Le llamamos "indexicalidad situada" porque este tipo de cognición normativa es altamente contextual y requiere del reconocimiento implícito de que cierta descripción le es aplicable a uno mismo (como en el ejemplo clásico de John Perry en donde uno sigue a la persona que está regando azúcar y descubre que es uno mismo). ${ }^{1}$ Aunque este tipo de cognición es suficiente para la eficacia del derecho, no es suficiente para el reconocimiento, adjudicación y cambio que necesitan procesos conscientes y deductivos.

Dado que los contextos epistémicos y morales utilizados por los sujetos de las normas son los que usan en su vida diaria, el gran misterio de la relación entre la moralidad y el derecho desaparece o al menos debe reconsiderarse seriamente desde nuevos supuestos. De igual manera, el de la eficacia normativa de un gran número de normas que sólo los abogados comprenden después de arduo estudio, pero que también son seguidas por el resto de los destinatarios de las mismas sin ese conocimiento también desaparece. Estas son dos ventajas de nuestra propuesta.

La analogía lingüística es fundamental. Estructura y sintaxis son inconscientes. Los aspectos normativos también son inconscientes. Uno requiere de lingüistas y psicólogos para entender explícitamente las normas gramaticales y del comportamiento. De igual manera, uno requiere de jueces para entender las normas jurídicas explícitamente. Sin embargo, uno no necesita de esos conocimientos necesariamente para ser un hablante competente.

La estrategia que seguimos en el artículo consta de dos partes: en primer término analizaremos el impacto de nuestra propuesta a un nivel meta-teórico, es decir, en las distintas tradiciones de teoría ge-

1 Perry, John, "The Problem of the Essential Indexical", Noûs, vol. 13, 1979, pp. 3-21. 
neral del derecho donde suscribimos un pluralismo metodológico; en segundo lugar nos ocupamos del papel de la indexicalidad como herramienta teórica para explicar nuestras prácticas jurídicas.

\section{PluRALISMo MEtódico, TRAdiciones EN FILOSOFÍA DEL DERECHO Y SITUACIONISMO}

Es ampliamente conocida en la metodología de la investigación científica la importancia de la relación entre el problema a resolver y la elección de las variables relevantes.

Esta decisión tiene importantes implicaciones en la selección del marco teórico, la metodología, e incluso en el tipo de matemáticas y recursos computacionales requeridos para su modelado, simulación y validación.

Por ejemplo, en el terreno de la física sabemos que el esfuerzo requerido para acelerar un objeto se expresa mediante la ecuación lineal: fuerza = masa $\mathrm{x}$ aceleración, cuya representación gráfica corresponde a una línea recta ascendente.

Dado que no es posible (o al menos no lo ha sido y no tenemos razones para suponer que algún día lo será) representar todo cuanto es el caso en el mundo en un solo modelo, el trabajo científico implica selección.

De esta manera, en la ecuación anterior se han considerado únicamente las dos variables dependientes "masa” y “aceleración”, pero todos sabemos que en el mundo también opera la fricción y que ésta cambia la magnitud del esfuerzo requerido para el desplazamiento del objeto y, por tanto, su velocidad.

Incluir la fricción como variable implica serias complicaciones que no pueden ser abordadas desde una ecuación lineal y cambia el tipo de matemáticas al de las ecuaciones no lineales.

Que un modelo deje de considerar ciertas variables asociables a su objeto de explicación no implica necesariamente su evaluación negativa a condición de que efectivamente dé cuenta del fenómeno que pretende explicar. Por ejemplo, Galileo explicó adecuadamente que dos cuerpos caen al mismo tiempo independientemente de la diferencia de sus masas sin considerar, precisamente, la fricción. 
Los factores que pueden influir en las decisiones metodológicas durante el diseño de una investigación científica pueden ser múltiples. Uno de particular importancia es el grado de desarrollo del conocimiento científico o tecnológico en un dominio específico y en un tiempo determinado. Así, el hecho de contar con súper computadoras hace posible abordar (y en última instancia definir) problemas inimaginables en el siglo XIX. Sin embargo, la elección de los elementos constitutivos del objeto de explicación (explanans) constituye una decisión fundamental para la cual no hay garantías metodologícas y frecuentemente queda a la sagacidad del científico.

Una importante diferencia entre las teorías científicas y las filosófico-jurídicas (así como con todas las demás teorías puramente conceptuales) es que éstas carecen de un estándar de verificación empírica para su validación. No obstante, elaborar una teoría jurídica también implica una selección de las propiedades relevantes del objeto de explicación o explanans, que se traduce en la o las preguntas que el teórico buscará responder.

Esto es lo que parece tener en mente Julie Dickson cuando presenta su concepto de proposición evaluativa indirecta referido a la teoría o filosofía del derecho:

An indirectly evaluative proposition of the form ' $\mathrm{X}$ ' is an important feature of the law" is thus a proposition which attributes some evaluative property to that of the law but which does not entail a directly evaluative proposition that the features of the law in question is good (or bad) ...In asserting that ' $\mathrm{X}$ is an important feature' we are accounting the existence of some $\mathrm{X}$ as significant and hence worthy of explanation. ${ }^{2}$

La cuestión que ahora surge respecto de la teoría del derecho es si podemos identificar algún criterio para determinar cuándo una propiedad requiere ser explicada y cuándo no. La respuesta a este problema es relevante porque se liga de manera directa con la evaluación de la virtud epistémica de la cobertura explicativa de una teoría (¿explica lo que debería explicar?).

2 Dickson, Julie, Evaluation and Legal Theory, Portland, Hart Publishing, 2001, p. 53. 
Paradójicamente, la respuesta a esta evaluación puede ser más compleja en el terreno de las teorías del derecho (y conceptuales en general) que en el de la ciencia, debido a la carencia de los mecanismos de autocorrectividad empírica que posibilitan corroborar las proposiciones predictivas que validan una teoría en el terreno científico.

Siguiendo esta línea de pensamiento, el pluralismo en las teorías jurídicas puede explicarse como diferentes instancias de la "X" en la fórmula de Dickson. Cada instanciación puede considerar una propiedad diferente del derecho como relevante para ser explicada y constituir el punto de partida de una tradición en la filosofía del derecho.

Esta caracterización del pluralismo metodológico en la teoría jurídica es coherente con el pensamiento del profesor Bix cuando dice que:

Legal systems are extremely complex. Inevitably, a theory about law can capture only a portion of the relevant facts...Once one accepts the importance of selection in constructing social theories, the focus then turns to the basis on which selection occurs. ${ }^{3}$

Además de la diversidad de propiedades relevantes, determinantes de distintas clases de problemas, la construcción de diferentes teorías jurídicas es guiada por la elección hecha entre una diversidad de métodos que hacen posible obtener inferencias confiables y la satisfacción exitosa de ciertos estándares epistémicos (coherencia, estabilidad, alto grado de invulnerabilidad a la contra-argumentación) y de esa manera proporcionar respuesta a los problemas originalmente planteados.

En síntesis: la relación entre la selección de cierto tipo de propiedades relevantes como dignas de ser explicadas, los problemas que de dicha selección surgen y la metodología seguida para afrontarlos producen diferentes teorías y tradiciones en la filosofía del derecho.

Por lo que respecta al objetivo central de este trabajo, la referencia al pluralismo metódico y la diversidad de tradiciones en la teoría

${ }^{3}$ Bix, Brian, Jurisprudence, Theory and Context, Carolina Academic Press, 2006, p. 4. 
del derecho resulta de suma relevancia, toda vez que cada una de ellas puede presuponer idealizaciones distintas acerca de las capacidades cognitivas de los sujetos de derecho y, por tanto, ser susceptibles de diferentes refutaciones a partir de los resultados de la investigación experimental. Con base en esto, debe resultar obvio que las capacidades cognitivas presupuestas por un ius-naturalista que considera que el derecho natural se conoce por revelación divina son diferentes a las de un defensor del análisis económico del derecho que suscribe la teoría de la decisión racional, o a la de un representante del realismo (o neo realismo) americano, para quien las decisiones judiciales obedecen básicamente a factores subjetivos, o a la de un analítico oxoniense que asume que el derecho es una práctica social. Como hemos señalado precedentemente, el objetivo de este trabajo es meramente exploratorio y por tanto no habremos de analizar a profundidad las capacidades cognitivas presupuestas por cada tradición y sus refutaciones. En su lugar nos limitaremos a realizar algunas consideraciones generales sobre la tradición analítica, el análisis económico del derecho y el (neo) realismo defendido por Leiter. Como se mostrará más adelante, resulta sorprendente percatarse de que, a pesar de sus diferencias, es posible encontrar una zona de intersección en los presupuestos de las diferentes tradiciones filosóficojurídicas que corresponde a la idealización cognitiva del destinatario del derecho como un agente racional, consciente del contenido de las normas jurídicas que debe seguir y que decide su comportamiento con base en la autonomía de su voluntad. Estos mismos presupuestos también son compartidos por otro tipo de teorías conceptuales de gran importancia en los sistemas jurídicos de tradición romano-germánica: las teorías doctrinales o de dogmática jurídica características de cada una de las diferentes áreas del derecho.

En todo caso, debe subrayarse que no es nuestro objetivo realizar un análisis detallado de las idealizaciones cognitivas en cada una de las referidas teorías, sino señalar el tipo de investigación que creemos debe caracterizar a un programa de naturalización en la filosofía del derecho, mismo que puede resumirse en la siguiente prescripción: aumentar el estándar de validación epistémica de las teorías jurídicas de tal suerte que vaya más allá del ejercicio dialéctico de la argumentación especulativa y llegue a ser una continuación 
de los resultados de la investigación en ciencias cognitivas, en un sentido semejante tiene lugar en el terreno de la filosofía de la mente contemporánea, donde ya no es posible hablar de homúnculos.

Por otra parte, las idealizaciones cognitivas en el derecho no quedan únicamente en el terreno de los presupuestos de los teóricos o filósofos del derecho, sino que también están presentes en los operadores jurídicos y, en general, en los demás destinatarios del derecho.

\section{IDEALIZACIÓN COGNITIVA (EL ENFOQUE PRESCRIPTIVO) VS. NATURALIZACIÓN EN LA FILOSOFÍA DEL DERECHO (EL ENFOQUE DESCRIPTIVO COMO CONDICIÓN DEL PRESCRIPTIVO)}

¿Cómo es posible la identificación personal de uno mismo como sujeto de cierta norma? Este problema tiene dos aspectos. El primero es normativo: ¿qué procesos cognitivos permiten seguir una regla? El segundo es semántico: ¿qué procesos cognitivos permiten el reconocimiento de una descripción como aplicable a uno mismo? A este último proceso se le puede llamar indexicalidad normativa.

Respuestas a la primera pregunta, que es de carácter normativo, son de dos tipos: cognición idealizada y cognición implementada. La cognición idealizada busca principios formales, como las reglas basadas en los axiomas de probabilidad para condicionar las creencias. Este es el tema de la epistemología formal. En contraste con esta propuesta formal, está la evidencia psicológica que demuestra que en muchos casos estos principios formales no son seguidos por los sujetos. La propuesta descriptiva busca encontrar cómo los agentes cognitivos razonan, no cómo deben razonar.

La evidencia en ciencia cognitiva demuestra que en varias circunstancias los agentes cognitivos no siguen reglas básicas de racionalidad idealizada. Por ejemplo, en varias situaciones los sujetos violan axiomas básicos de la probabilidad, en contra de los principios asumidos en la epistemología formal Bayesiana. Dichos resultados han sido interpretados como muestra de irracionalidad en la toma de decisiones bajo incertidumbre en la psicología humana. En psicología esto ha conducido a un debate entre teóricos que señalan que la irracionalidad es inevitable y teóricos que niegan que la teoría idea- 


\section{ENRIQUE CÁCERES / CARLOS MONTEMAYOR}

lizada de la racionalidad sea la adecuada, argumentando que, por el contrario, dichos resultados muestran que la racionalidad humana es flexible, contextual y heurística. ${ }^{4}$ Este debate tiene implicaciones que son centrales para el tema de la eficacia del derecho. Si los seres humanos no pueden seguir reglas de manera consistente y racional (tanto en ética y en derecho como en epistemología), el cumplimiento de las normas que las teorías del derecho asumen es un mito o un misterio. Argumentaremos que la base de este problema radica en que los teóricos del derecho no han distinguido adecuadamente los dos procesos cognitivos que mencionamos anteriormente: indexicalidad consciente e inconsciente o implícita.

En filosofía hay dos vertientes de crítica situacionista, que favorecen la tesis escéptica. Una es de carácter moral y la otra es de carácter epistémico. Gilbert Harman y John Doris señalan que la evidencia científica demuestra que las virtudes éticas no tienen sustento porque no hay disposiciones suficientemente robustas para calificar como virtudes. Lo que hay son disposiciones que dependen demasiado de la situación en la que los sujetos deben responder: de ahí el término situacionismo. ${ }^{5}$ Nótese que esta es una crítica dirigida principalmente a las virtudes. En epistemología han habido críticas similares, basadas en el trabajo de Kahneman y Tversky, ${ }^{6}$ pero el situacionismo epistémico es mucho menos plausible que el ético. ${ }^{7}$ No obstante la implausibilidad del situacionismo epistémico, es muy

4 Kahneman, Daniel, Thinking Fast and Slow, Nueva York, Farrar Straus and Giroux, 2011 y Gigerenzer, Gerd, Rationality for Mortals: How People Cope with Uncertainty, Nueva York, Oxford University Press, 2008.

5 Harman, Gilbert, "Moral Philosophy Meets Social Psychology: Virtue Ethics and the Fundamental Attribution Error", Proceedings of the Aristotelian Society, nueva serie, vol. 119, 1999, pp. 316-331; Harman, Gilbert, "The Nonexistence of Character Traits", Proceedings of the Aristotelian Society, vol. 100, núm. 1, 2000, pp. 223-226; Doris, John M., Lack of Character: Personality and Moral Behavior, Cambridge, Cambridge University Press, 2002.

6 Kahneman, Daniel y Tversky, Amos, "On the Psychology of Prediction”, Psychological Review, vol. 80, núm. 4, 1973, pp. 237-251.

7 Morton, Adam, Bounded Thinking, Oxford University Press, 2012; Fairweather, Abrol and Montemayor, Carlos, "Epistemic Dexterity: a Ramseyian Account of AgentBased Knowledge", en Fairweather, Abrol y Flanagan, Owen (eds.), Naturalizing Epistemic Virtue, Nueva York, Cambridge University Press, 2014. 
importante analizar la propuesta de Kahneman para apreciar la distinción que proponemos entre indexicalidad consciente o reflexiva e indexicalidad implícita. Es cierto que gran parte de nuestras capacidades cognitivas son situadas y contextuales, pero no se sigue de esto que seamos incapaces de seguir normas jurídicas, epistémicas y morales.

En el caso de la ética, la crítica situacionista es más plausible. No sólo se aplica a teorías de la virtud ética, sino a teorías que tienen presupuestos cognitivos que la evidencia empírica ha demostrado son muy ambiciosos. Por ejemplo, Stich criticó los presupuestos kantianos de la teoría de Rawls y del equilibrio reflexivo en general. La noción de que los sujetos de las normas tienen, conscientemente y con esfuerzo cognitivo, que reflexionar sobre las implicaciones de un precepto para seguirlo como si fuera una inferencia lógica está muy arraigado a las teorías del derecho. El tribunal de la razón consciente es un mito que la evidencia científica ha falsificado. Pero aunque los sujetos de las normas no requieren de dichos procesos conscientes y deductivos para actuar, los jueces y abogados sí lo requieren. Si uno acepta literalmente la crítica situacionista, pareciera ser que cualquier requerimiento únicamente racional en la teoría del derecho debe ser rechazado, porque la teoría del derecho asume principios falsos sobre la racionalidad humana. Pero hay otra alternativa, que es la que defendemos. Procesos como el equilibrio reflexivo sólo son aplicables a operadores jurídicos como jueces, abogados y demás funcionarios encargados de aplicar el derecho.

A nadie más se le puede exigir dicho razonamiento consciente. A diferencia de nuestras actividades diarias, los jueces y abogados deben razonar de modos similares al equilibrio reflexivo. Esto es compatible con la evidencia empírica, incluso con la interpretación escéptica de Kahneman, como mostramos a continuación.

Kahneman argumenta que existen dos sistemas de razonamiento, o mejor dicho, dos formas de razonamiento analógicamente similares, que clasifica como los sistemas 1 y 2 . El sistema 1 es rápido, flexible, muy contextual y no confiable. Este sistema produce los problemas documentados en la evidencia experimental en los que sujetos violan axiomas básicos de la probabilidad, dependiendo de la información que les es presentada antes de contestar. El sistema 2, 
por el contrario, es lento, requiere de atención consciente y voluntaria, pero al mismo tiempo es "flojo," y "deja” que el sistema 1 tome las riendas en la mayoría de los casos. El sistema 2, sin embargo, es muy confiable. Éste es el sistema que los matemáticos usan cuando prueban o deducen verdades matemáticas y es el que usamos cuando queremos seguir paso a paso un argumento. El sistema 1, Kahneman dice, casi siempre gana la batalla en contra del sistema 2. Nuestro cerebro, de acuerdo con Kahneman, está configurado de modo que el sistema en el que dependemos para navegar la información que confrontamos a diario es el sistema 1. Aunque debemos, idealmente, implementar el sistema 2 , muy raramente lo hacemos. ${ }^{8}$

Como mencionamos anteriormente, esta interpretación de los resultados científicos no es incuestionable (véase Gigerenzer). ${ }^{9}$ Pero incluso usando la interpretación escéptica de Kahneman, existe la posibilidad de rescatar la racionalidad en el derecho sin contradecir los resultados empíricos. Para esto necesitamos de tres analogías lingüísticas.

Primero, hacemos la distinción que hace Perry entre entender que una descripción le aplica en la tercera persona y entender que la descripción le aplica a uno en la primera persona. En su ejemplo, uno puede seguir a un sujeto que está haciendo un reguero de azúcar en un centro comercial y pensar que dicho sujeto es descuidado. El contenido de esta proposición cambia radicalmente cuando uno se da cuenta que el que está haciendo el reguero es uno mismo. La proposición no cambia de contenido en la descripción de la acción, pero cambia en la perspectiva del sujeto, y tiene consecuencias importantes para la acción. Creemos que este tipo de contenido indéxico es fundamental en la caracterización del sistema 1 de Kahneman, que puede ser inconsciente, flexible, contextual e implícito. Algo similar ocurre entre los sujetos de las normas que siempre actúan en un contexto y los jueces y abogados que deben razonar sobre lo que unifica a varios contextos normativos.

La siguiente analogía tiene que ver con el conocimiento de la sintaxis del lenguaje. Los hablantes de una lengua saben perfectamente

8 Kahneman, Thinking Fast and Slow, cit.

9 Gigerenzer, op. cit. 
la sintaxis de esa lengua (la distinción entre sujeto, verbo, adverbio). Dichos hablantes usan este conocimiento de manera inconsciente e implícita ${ }^{10}$ cuando hablan (sistema 1), pero los lingüistas que dedican su vida a entender la sintaxis de una lengua usan razonamiento consciente y explícito para formular las reglas del lenguaje que estudian (sistema 2). Algo similar ocurre entre los sujetos de las normas que las siguen implícitamente y los jueces y abogados que las estudian explícitamente.

Finalmente, existe una analogía semántica con la división de la labor lingüística de Putnam. ${ }^{11}$ Sin conceder sus tesis externalistas, y usando la analogía sintáctica, uno puede apreciar que el contenido de las normas es estudiado y entendido sólo por un subconjunto de los miembros de un sistema normativo: los operadores jurídicos como los jueces y abogados. El resto de la comunidad depende del entendimiento explícito, voluntario y consciente de este subconjunto de individuos para garantizar que, entre otras cosas, el sistema jurídico que los gobierna sea consistente.

Como hemos señalado precedentemente, proponemos que la indexicalidad cognitiva situada (relacionada con el problema del marco) puede ser consciente o inconsciente. La evidencia relevante es la investigación de heurísticas para la decisión. La tesis fundamental que defendemos es que aun cuando la cognición consciente que permite el cálculo racional durante la aplicación de las normas es, obviamente, suficiente, para el seguimiento del orden normativo, dicho tipo de cognición no es necesaria. Es sólo necesaria para dichos operadores y aquellos que deben seguir explícitamente la relación entre preceptos legales y casos concretos y cuyo trabajo es aplicar las normas del sistema. Nótese, sin embargo, que aunque la cognición consciente es suficiente para el seguimiento de normas, ésta no ofrece un modelo realista de la eficacia normativa, uno ja-

10 Chomsky, Noam, Knowledge of Language, Nueva York, Praeger, 1986; Chomsky, Noam, "Generative Grammar: Its Basis, Development and Prospects", Studies in English Literature and Linguistics, Kyoto, Kyoto University, 1987.

11 Putnam, Hilary, “The Meaning of 'Meaning'”, en Gunderson, Keith (ed.), Language, Mind and Knowledge, Minnesota Studies in the Philosophy of Science, University of Minnesota Press, 1975, vol. VII; reprinted in Putnam, Hilary, Mind, Language and Reality, Philosophical Papers, Cambridge University Press, 1975, vol. 2. 
más piensa en las regulaciones sobre compra-venta cuando paga un boleto del metro.

\section{LA IDEALIZACIÓN COGNITIVA Y EL PRESUPUESTO DE LA EFICACIA JURÍDICA EN LAS DISTINTAS TRADICIONES FILOSÓFICO-JURÍDICAS}

Como hemos señalado, la selección de los elementos constitutivos del explanans es una decisión metodológica fundamental en la teoría jurídica. Igualmente afirmamos que uno de los presupuestos fundamentales de la teoría jurídica y del derecho en general es que éste regula la vida social. En lo sucesivo nos referiremos a este presupuesto con "presupuesto de la eficacia normativa", o simplemente "P.E.J."

A pesar de su innegable importancia y por tanto constituir un "X" que merece ser explicado, el P.E.J no ha sido objeto de análisis teórico por parte de las principales teorías analíticas del derecho.

La importancia de dar una explicación satisfactoria del P.E.J se pone de manifiesto en preguntas del tipo: ¿en qué consiste el P.E.J. y cómo se justifica?, ¿cómo opera el derecho cuando genera conductas (y prácticas social[es])?, ¿cómo se incorporan las normas jurídicas en los procesos que culminan en una conducta determinada?, ¿operan de manera diferente a como lo hacen normas de otro tipo?, ¿en qué grado las normas jurídicas son efectivamente razones para la acción?, ¿en qué medida los individuos observan conductas que corresponden a la exigencia normativa por razones diferentes a las normativas jurídicas?, ¿una vez incorporadas por el destinatario de la norma adquieren estatus de creencias?, ¿qué dinámica de derrotabilidad opera o debe operar con creencias diferentes?

\section{La idealización cognitiva en la filosofía analítica}

En otra parte de este trabajo hemos sostenido que una buena manera de abordar un situacionismo jurídico sería partir de la reacción que el situacionalismo ético representa para la ética de virtudes.

No obstante, es necesario tener presente una variable de gran significación que distingue al situacionismo ético del jurídico: la separación entre derecho y moral. 
Esto implica que, a diferencia de lo que ocurre con la moral, el derecho es el producto de las propiedades constitutivas de las normas jurídicas y no un producto cultural adquirido por aprendizaje vicario o que venga pre-programado en nuestro cableado neuronal. En buena medida, es una construcción artificial.

En este sentido, las normas del derecho son equivalentes a las reglas constitutivas de Searle y los hechos jurídicos a sus hechos institucionales.

Trataremos de ilustrar lo anterior mediante el siguiente ejemplo: supongamos el gobierno de un país que necesita recursos extraordinarios para hacer frente a un déficit financiero y en un estado de cosas 1 promulga una ley que establece un impuesto sobre la tenencia de ventanas. Durante el tiempo en que dicha norma es vigente, es posible identificar en el mundo de los hechos institucionales conductas correspondientes al delito de evasión del pago del impuesto sobre ventanas. Una vez resuelta la crisis financiera se deroga la norma que establecía al impuesto sobre ventanas. En este segundo estado de cosas, es imposible encontrar en el mundo de los hechos institucionales conductas correspondientes al delito de evasión del pago de impuesto sobre ventanas. Desde luego en el caso de la moral no existe un equivalente a la función constitutiva del derecho.

En lo siguiente asumiremos que la constitutividad jurídica es otro presupuesto central de las teorías del derecho.

\section{El P.E.J. y la idealización cognitiva en Austin, Hart y Kelsen}

Como es bien conocido, para John Austin el derecho se caracteriza por cierto tipo de mandatos emitidos por el gobernante a sus súbditos con la posibilidad de infringirles castigos en caso de que no lo obedezcan. Para él, la conducta de obediencia al derecho está caracterizada por el temor al castigo.

A partir de los problemas de la teoría austiniana, Hart elabora una teoría más sofisticada en la que la conducta de los sujetos de derecho no obedece únicamente al miedo a las sanciones, sino también a la consecución de objetivos lícitos, base de su distinción entre reglas primarias y reglas secundarias. A diferencia de Austin, la regla de 
reconocimiento es el resultado de prácticas institucionales y no un producto volitivo del rey.

Por otra parte, cabe resaltar que mientras Austin arroja luces fundamentalmente sobre la incidencia del derecho en los ciudadanos, Hart lo hace sobre los oficiales.

A pesar de que en estricto sentido no suele ser considerado un analítico, para los efectos de este trabajo incluimos en este rubro a Kelsen en tanto tiene finalidades semejantes a las de los autores anteriores y metodológicamente comparte con ellos el análisis conceptual.

A pesar del estricto formalismo con el que Kelsen pretende caracterizar al derecho, reconoce que la existencia de un sistema normativo depende de su eficacia. Es decir, un sistema jurídico existe si es eficaz y para ser eficaz es necesario que se realicen las conductas por las que se crean las normas del sistema, conforme a las reglas de producción normativa que el propio sistema establece. Éstas, a su vez, son condición necesaria para que la conducta jurídica de la sociedad en general pueda tener lugar.

Desafortunadamente ninguno de estos autores explicita las capacidades cognitivas de los destinatarios de la norma presupuestas para que el derecho sea eficaz. Sin embargo, la estructura de sus teorías es compatible con el siguiente modelo que recoge las intuiciones comunes acerca del derecho.

Los destinatarios de las normas:

1) Conocen los enunciados que forman parte del sistema jurídico (con base en cierta teoría de las fuentes) y tienen la capacidad de integrar su contenido como parte del sistema de creencias que rige su conducta.

De no ser así ¿qué sentido tendría que un rey o parlamento emitieran mandatos o reglas, sostener que la existencia de un sistema jurídico depende del cumplimiento de sus normas, o que éstas se distinguen de órdenes respaldadas por amenazas por su aspecto interno?

2) Dichas creencias normativas tienen una estructura constante que hace posible respetarlas independientemente de factores contextuales. 
Este postulado está en la base de la vinculatoriedad del derecho y en considerar a las normas jurídicas como razones protegidas que pretenden derrotar cualquier razón en contra de su observancia.

3) El comportamiento esperado a partir de las referidas creencias normativas está sujeto a mecanismos psicológicos de autorregulación.

Este postulado da cuenta de uno de los objetivos básicos del derecho consistente en modificar la dinámica cognitiva de sus destinatarios a efecto de que sean ellos mismos quienes ajusten su conducta a la prescripción normativa, aun cuando tengan que reprimir otras conductas.

4) Tienen control consciente sobre los mecanismos aludidos en “3)" y por tanto deciden también conscientemente si actúan o no de conformidad con las creencias referidas en "1)".

Es una condición necesaria para la racionalidad del derecho y está en la base incluso de aquellos casos en que se decide deliberadamente no obedecer el derecho como acontece en el caso de la desobediencia civil.

5) Respetar o no la norma es una cuestión de voluntad.

Está íntimamente vinculado con la propiedad anterior y queda ejemplificada con aquellos casos en que se decide entre cumplir con el pago de impuestos o generar estrategias de evasión fiscal, o entre robar o no un automóvil.

\section{Constitutividad jurídica e indexicalidad}

Una función principal de la constitutividad jurídica es la creación de diferentes "clases de sujetos normativos" (contribuyente, comprador, vendedor, arrendatario, homicida, defraudador, etcétera) cada uno de los cuales debe observar las conductas correspondientes atribuidas por el derecho.

En la práctica, una persona puede instanciar diferentes sujetos normativos y por tanto estar en posibilidad u obligación de realizar determinadas conductas para obtener o evitar ciertos resultados.

En congruencia con la idealización referida, esto significaría asumir que cada persona está consciente del papel jurídico que le toca 
ENRIQUE CÁCERES / CARLOS MONTEMAYOR

representar en cada momento y decide consciente y voluntariamente su comportamiento jurídico.

\section{Crítica a la idealización de estos teóricos}

Como hemos indicado precedentemente la naturalización filosófica implica un incremento en el estándar de validación de las teorías más allá de la dialógica conceptual y exige que al menos sean compatibles con los resultados de la investigación científica. Algo semejante a lo que ocurre con las actuales teorías de la filosofía de la mente que no pueden ignorar los avances de las neurociencias.

A continuación sometemos a ese test las propiedades del modelo idealizado de las capacidades cognitivas de los destinatarios de las normas que fundamentan el supuesto de la eficacia jurídica entre los positivistas:

1) Cuentan con la información suficiente para integrar el contenido normativo (constitutivo) a su sistema de creencias.

Aunque no está directamente vinculado con la idealización cognitiva es importante desenmascarar el supuesto de que los destinatarios de las normas tengan acceso a los contenidos normativos que les atañen y por tanto se comporten en consecuencia. Dicho brevemente, el paso que va del emisor de la norma (el rey o el parlamento) al receptor ocurre excepcionalmente y en la mayoría de los casos la conducta social jurídicamente relevante se realiza sin incorporación de contenidos normativos.

Como ejemplo de soporte empírico de esta afirmación, podemos referir una investigación interdisciplinaria entre la psicología social y el derecho sobre las bases psicológicas del comportamiento jurídico en México realizada en la UNAM. Dicha investigación, realizada en cinco estados de la República mexicana acerca de normas de tránsito, permitió constatar el desconocimiento absoluto de las normas de tránsito por parte de quienes conducen cotidianamente en el país. Se mostró que las razones por las que se comportaban los sujetos de la investigación obedecían a consideraciones morales o simplemente a balances costo beneficio en los que ponderaban el costo del posible soborno a entregar al agente, contra la probabili- 
dad de quedar impunes por faltas de tránsito. El contenido normativo muy excepcionalmente formaba parte del sistema de creencias de los sujetos.

El hecho de que la investigación se hubiera realizado sobre algo tan cotidiano como la legislación de tránsito permite conjeturar el desconocimiento de normas más especializadas y por tanto la imposibilidad de auto-ubicación de los destinatarios de las normas en términos de indexicalidad jurídica.

Dicho brevemente, los destinatarios de las normas carecen del conocimiento de las categorías normativas (sujetos de derecho) que pueden instanciar y consecuentemente no pueden autosituarse como el modelo idealizado hace suponer. Simplemente no ocurre al acto de autoconsciencia expresable mediante: ¡las indicaciones de la patrulla son para mí debido a que yo soy quien acaba de infringir el reglamento de tránsito! Desde luego los supuestos cálculos conscientes y racionales involucrados en la decisión de si realizo una conducta contraria a un norma de tránsito o no, es más irreal todavía.

Por otra parte, no debe asumirse que la no indexicalización a partir de las normas jurídicas es necesariamente una cuestión de conocimiento o desconocimiento de las mismas. Al igual que ocurre con un ciudadano, un jurista no entra a un restaurante pensando: ahora soy un comensal que está celebrando un contrato de prestación de servicios conforme al artículo "x" del Código de Comercio, de igual manera que el mesero no se estará autoubicando como trabajador en los términos de la Ley Federal del Trabajo.

Evidentemente lo anterior no implica sostener que el derecho no juegue ningún papel en la regulación de la conducta social, sino simplemente que su eficacia no opera en términos del modelo idealizado expuesto. ¿Qué teoría naturalizada puede explicar adecuadamente el presupuesto de eficacia jurídica? Es una pregunta abierta para la nueva línea de investigación que estamos explorando.

2) Dichas creencias normativas tienen una estructura constante e invariantista que debe respetarse independientemente de factores contextuales.

Esta propiedad, semejante a la que tiene lugar en las diversas propuestas de la ética de la virtud, recuerda al universalismo kantiano y queda sujeta a refutaciones semejantes a las que se dirigen por 
parte del situacionismo ético. Se ha mostrado experimentalmente que las creencias normativas que pueden dirigir nuestra conducta son inestables, dependientes de contexto y que factores éticamente irrelevantes como puede ser tener prisa o no para llegar a una cita puede marcar la diferencia entre ser solidario con alguien que solicita ayuda o no. En este sentido, la supuesta estabilidad de las creencias normativas del derecho es un presupuesto no justificado que está íntimamente ligado con la conocida expresión latina Dura lex sed lex, fuente de una de las críticas más arraigadas en contra del positivismo: el formalismo extremo.

3) Poseen mecanismos de autorregulación de las conductas que realizarán a partir de sus creencias normativas.

Una vez más se presenta una analogía con la ética de la virtud y los conceptos de conciencia e inconsciencia.

Si la incorporación de las normas al sistema de creencias es una condición de esta propiedad, resulta claro que su actualización es poco frecuente.

Sin embargo, aun asumiendo la integración de las normas, como podría ser el caso de un oficial, muchos de los resultados obtenidos en el campo del inconsciente adaptativo muestran que en un altísimo porcentaje, nuestras decisiones aparentemente conscientes son estados emergentes de procesos inconscientes sobre los que no tenemos el control supuesto en "2)".

4) Respetar o no la norma es una cuestión volitiva.

En este punto traemos a colación lo referido previamente respecto de los experimentos que muestran la ilusión de la voluntad.

Para concluir con este apartado deseamos referirnos a dos cuestiones importantes que invitan a la reflexión naturalizada: la primera se expresa en la pregunta: ¿en qué grado la supuesta regulación normativa del derecho es un epifenómeno de prácticas sociales que de una u otra forma tendrían lugar sin él? A fin de cuentas, la propiedad, las transacciones comerciales y los matrimonios existen mucho antes que cualquier código civil, legislación mercantil o el concepto técnico de "atributo de la personalidad". El hecho de que esas conductas acontezcan en la vida de Juan Pérez sin la exigencia de que él pueda auto-situarse conscientemente en el rol jurídico exi- 
gido por normas, equivale a decir que para él dichas prácticas (que) operan sin el derecho: simplemente son parte de la vida.

Por otra parte, la idealización del P.E.J. analizado plantea un dilema ético que tiene importantes consecuencias en la racionalidad del derecho: por una parte justifica mecanismos tales como la imposición de sanciones o la atribución de responsabilidad al asumir injustificadamente que el sujeto ha tenido conocimiento del deber impuesto por el sistema jurídico, y exigir su autosituación consciente en él. Mientras que en el otro polo se encuentra el hecho de que exigir (y no simplemente suponer) el conocimiento y autoubicación jurídicas haría inoperante al sistema, lo que se expresa en la conocida fórmula de dudosa justificación política y moral: "la ignorancia de la ley no exime del cumplimiento de la misma".

\section{La idealización cognitiva en el análisis económico del derecho}

Uno de los ámbitos en el que la naturalización ha tenido mayor impacto es el de la economía.

El modelo idealizado corresponde a la teoría de la decisión racional o agentes maximizadores.

A través de la economía experimental o nueroeconomics se ha mostrado que los agentes que deciden se comportan de la manera que predice dicha teoría.

El análisis económico del derecho constituye una tradición que se basa en la misma teoría idealizada y por tanto es objeto de las mismas críticas.

\section{Más allá de la filosofía del derecho: la doctrina jurídica y el derecho positivo}

Hasta este momento hemos centrado nuestras reflexiones en teorías generales del derecho, sin embargo creemos que el tipo de consideraciones realizadas pueden extenderse a otros ámbitos de la construcción teórico-jurídica: a las teorías particulares o doctrinas de la dogmática jurídica. En ellas también es posible encontrar idea- 
lizaciones de las propiedades cognitivas de los destinatarios de las normas que no soportarían el análisis de la naturalización.

Llama la atención el hecho de que, al igual que acontece con las diversas tradiciones en filosofía del derecho, también en la doctrina es frecuente encontrar diversas tradiciones en torno a un mismo tema. Por ejemplo, la teoría del delito es desarrollada de manera distinta por la tradición causalista, la finalista, la lógico-matemática o la funcionalista y cada una de ellas puede estar presuponiendo idealizaciones cognitivas susceptibles de validación mediante naturalización filosófica.

Desde luego no es el espacio para desarrollar a profundidad este tipo de reflexión. En lugar de ello, procedemos a ejemplificarlo con una cita representativa del concepto de culpabilidad defendido por la llamada "Teoría normativa o normativista de la culpabilidad":

"Con el juicio desvaloratio de la culpabilidad, se reprochará al autor el que no ha actuado conforme a Derecho, él se ha decidido a favor del injusto, aun cuando podría comportarse conforme a Derecho, aún cuando podía decidirse a favor del Derecho".

Como es fácil observar en esta cita de Reinhart Maurach ${ }^{12}$ los suscriptores de dicha teoría adoptan el mismo modelo idealizado desarrollado al abordar las teorías de Austin, Hart y Kelsen. Es notorio que los autores asumen que la culpabilidad presupone la incorporación de la norma penal a su sistema de creencias, el control consciente de mecanismos de autorregulación de la conducta y una decisión de actuar en contra de lo establecido por la norma mediante un acto volitivo.

El carácter "teórico" de esta conceptualización puede tener una gran trascendencia práctica, pues si es adoptado por un juez penal como criterio de interpretación del significado de la palabra "culpabilidad" contenido en la legislación traerá aparejados serios problemas epistemológicos para determinar la verdad o falsedad de la afirmación: "X" es responsable de la conducta ilícita "C", pues requerirá probar que el sujeto conocía la norma al momento de cometer el ilícito y que voluntariamente decidió actuar en contra de lo que

12 Castellanos Tena, Fernando, Lineamientos elementales de derecho penal, México, Porrúa, 1977, p. 234. 
ella establecía. Evidentemente probar proposiciones expost-fácticas aseverativas de estados mentales frecuentemente presenta dificultades insuperables.

Desde luego que la situación se empeora si en lugar de constituir un criterio de interpretación, el concepto de culpabilidad referido se positiviza en un artículo del código penal.

Esto significa que la validación conceptual por vía de naturalización puede ir más allá del ejercicio académico y plantea importantes reflexiones en torno al derecho positivo y la teoría de la legislación: ¿en qué medida el derecho se basa en modelos de idealización cognitiva que serían refutados por la investigación experimental?

\section{CONCLUSIÓN}

Hasta este punto hemos expuesto razones en contra de la idealización cognitiva presupuesta tanto por los teóricos del derecho (razón teórica) como en las representaciones sociales acerca del mismo consistente en una sobrevaloración de la función del control consciente y una especie de metacognición continua que rigen la conducta social.

Esta refutación podría dar la impresión de que se niega totalmente el papel del control consciente, lo que resultaría contraintuitivo con las creencias tan arraigadas en nuestra manera de comprender la conducta humana y la manera de "modelarla" a través del derecho.

La tesis que suscribimos en este trabajo no niega la existencia e importancia de los procesos conscientes, ni tampoco sostiene que todo nuestro aprendizaje conductual sea únicamente vicario.

Sin embargo, lo que sí sostenemos es que la forma en que las idealizaciones cognitivas referidas deben ser sustituidas por explicaciones naturalizadas que den cuenta de la interacción entre los niveles consciente e inconsciente como capas subemergente y emergente de un mismo sistema. Algo semejante a los sistemas 1 y 2 de Kahneman.

Una explicación basada en la relación entre ambos niveles del mismo sistema cognitivo resulta de gran importancia para respon- 
der a dos preguntas fundamentales que hasta la fecha han pasado desapercibidas tanto para la teoría del derecho como para nuestras representaciones sociales acerca del mismo: si las normas jurídicas entendidas como las proposiciones contenidas en las fuentes jurídicas son desconocidas por parte de los ciudadanos, entonces ¿en qué sentido el derecho regula nuestra vida social en general? Y, por otra parte, si el derecho opera únicamente a nivel inconsciente sobre los que no tenemos control, ¿cómo explicar los procesos de razonamiento que tienen lugar entre dos litigantes cuyos procesos cognitivos son orientados por cierta teleología, o los realizados por los jueces al momento de ponderar las pruebas ofrecidas por las partes?

La respuesta que damos a estas cuestiones está en los dos sistemas referidos y en dos tipos de indexicalidad situacional que hemos dado en llamar "implícita” y "explícita”.

La indexicalidad situacional implícita tiene lugar cuando los ciudadanos se comportan conforme a las expectativas de las normas jurídicas simplemente por encontrarse en ciertos contextos o situaciones. En estos casos, no es necesario que tengan el conocimiento explícito de las normas jurídicas y regulan su conducta generalmente por adaptación a las interacciones sociales con oficiales encargados de la ejecución del derecho. Un ejemplo típico lo constituye la solicitud de un pasaporte, donde la racionalidad implícita en la documentación solicitada y que en algún sentido legitima a la legislación correspondiente, es absolutamente desconocida por el ciudadano medio. Éste simplemente obedece las indicaciones realizadas cara a cara por el funcionario en turno. En otras ocasiones, la conducta coincidente con lo establecido por las normas puede no requerir de la interacción con un oficial, sino tener lugar en "contextos abiertos". Esto es lo que sucede con alguien que se abstiene de robar un objeto, en contra de sus primeros impulsos. En estos casos, aunque la conducta coincide con las expectativas de las normas jurídicas, no podemos asumir que dichas normas han sido las razones para su acción.

Este fenómeno es de suma importancia dado que puede presuponer la activación de creencias no inducidas por las normas jurídicas, sino por normas morales y pone en tela de juicio el sentido de la diferencia tajante entre derecho y moral, o si la moral forma parte del 
concepto de derecho, en lugar de pensar en sistemas de creencias con zonas de intersección de creencias cognitivamente indisolubles.

Por otra parte, los oficiales también se sitúan como ciudadanos en determinados contextos (el ejemplo del jurista que asiste al restaurante). Sin embargo, en otros contextos opera una especie de "switcheo cognitivo" que produce una indexicalidad situacional que pasa a operar a nivel consciente y donde el procesamiento del contenido proposicional de las normas jurídicas es central. Para ilustrar esto volvamos al ejemplo del jurista que asiste al restaurante: imaginemos que se encuentra como cualquier comensal disfrutando de su platillo sin haberse situado en un nivel en el que codifica su entorno de términos jurídicos (no ve al mesero como un trabajador en términos del artículo "X" de la Ley Federal del Trabajo, ni al restaurante como un establecimiento constituido conforme a la Ley General de Sociedades Mercantiles, ni a él como parte en un contrato de prestación de servicios, etcétera). Como cliente frecuente del restaurante tiene cierto grado de familiaridad con el mesero que lo atiende a quien percibe con una energía bastante menor que la habitual y le pregunta por su estado de salud. El mesero admite no sentirse bien desde hace algunos días y el cliente le pregunta si ya fue al médico, ante la respuesta negativa del mesero debido al alto costo de las consultas privadas de los médicos. Acto seguido el cliente-jurista le pregunta por la seguridad social que debe brindarle el patrón, misma que, a decir del mesero, no le ha sido concedida. En ese momento el jurista, como reflejo natural comienza a dar una asesoría gratuita sobre los derechos a la seguridad social de los trabajadores. En este caso, el paso del sistema 1 al sistema 2 es dado cuando el abogado se percata de la falta de cumplimento de las obligaciones jurídicas de los patrones, de conformidad con la legislación y ha operado a nivel consciente en la elaboración de la asesoría. El switcheo ha dado lugar al paso de una indexicalidad situada implícita a una indexicalidad situada explícita propia de los operadores del sistema.

La diferencia entre ambos sistemas es crucial para comprender problemas fundamentales del derecho como el de la atribución de responsabilidad al suponer que los ciudadanos actúan con base en el sistema 2 y por tanto en una indexicalidad situacional explícita cuando ello no es así. 
Concluimos este trabajo volviendo a subrayar que no es nuestra intención (aún) presentar una propuesta teórica robusta, sino arrojar nuevas luces sobre problemas hasta la fecha ignorados en la teoría del derecho, que son semejantes a los que han sido en otros dominios filosóficos como la ética o la epistemología como consecuencia del impacto del giro cognitivo. Con ello intentamos sintonizar nuestras manera de comprender los fenómenos jurídicos con los nuevos paradigmas que están cambiando nuestra manera de comprender al mundo y, tal vez como nunca antes, a nosotros mismos.

\section{BIBLIOGRAFÍA}

Austin, John, The Province of Jurisprudence Determined, Nueva York, Burt Franklin, 1970.

BIX, Brian, Jurisprudence, Theory and Context, Carolina Academic Press, 2006.

CÁceres Nieto, Enrique y RodríGuez, Graciela, Bases psicológicas del comportamiento jurídico en México, México, UNAM, Instituto de Investigaciones Jurídicas, Facultad de Psicología, 2008.

Castellanos Tena, Fernando, Lineamientos elementales de derecho penal, México, Porrúa, 1977.

СномsкY, Noam, Knowledge of Language, Nueva York, Praeger, 1986.

- - - "Generative Grammar: its Basis, Development and Prospects", Studies in English Literature and Linguistics, Kyoto, Kyoto University, 1987.

Dickson, Julie, Evaluation and Legal Theory, Portland, Hart Publishing, 2001.

DoRIs, John M., Lack of Character: Personality and Moral Behavior, Cambridge, Cambridge University Press, 2002.

Fairweather, Abrol y Montemayor, Carlos, "Epistemic Dexterity: a Ramseyian Account of Agent-Based Knowledge", en FAIRWEATher, Abrol y Flanagan, Owen (eds.), Naturalizing 
Epistemic Virtue, Nueva York, Cambridge University Press, 2014.

GIGERENZER, Gerd, Rationality for Mortals: How people cope with uncertainty, Nueva York, Oxford University Press, 2008.

HARMAN, Gilbert, "Moral Philosophy Meets Social Psychology: Virtue Ethics and the Fundamental Attribution Error", Proceedings of the Aristotelian Society, nueva serie, vol. 119, 1999.

- - - "The Nonexistence of Character Traits", Proceedings of the Aristotelian Society, vol. 100, núm. 1, 2000.

HART, H. L. A., El concepto de derecho, trad. de Genaro Carrió, Argentina, Abeledo Perrot, 1961.

Kahneman, Daniel y Tversky, Amos, "On the Psychology of Prediction”, Psychological Review, vol. 80, núm. 4, 1973.

Kahneman, Daniel, Thinking Fast and Slow, Nueva York, Farrar Straus and Giroux, 2011.

Kelsen, Hans, La teoría pura del derecho, 2a. ed., trad. de Roberto J. Vernengo, México, Instituto de Investigaciones Jurídicas, 1982.

Morton, Adam, Bounded Thinking, Oxford University Press, 2012.

Putnam, Hilary, "The Meaning of «Meaning»", en Gunderson, Keith (ed.), Language, Mind and Knowledge (Minnesota Studies in the Philosophy of Science, vol. VII), Minneapolis, University of Minnesota Press, 1975; reimpreso en Putnam, Hilary, Mind, Language and Reality (Philosophical Papers, vol. 2), Cambridge, Cambridge University Press, 1975. 